\title{
Paracentesis: datos sobre la correcta utilización *
}

\section{Paracentesis, Appropriate Technique and Clinical Application}

Fecha de recepción: 19/01/2017 | Fecha de aprobación: 11/04/2017 | 30/01/2017

\author{
Gabriel Mosquera-KLINGer ${ }^{\mathrm{a}}$ \\ Hospital Pablo Tobón Uribe, Colombia \\ ORCID: http://orcid.org/0000-0002-3108-0424 \\ Melissa Mayo-Patiño \\ Fundación Santa Fe de Bogotá, Colombia \\ YANETT SuÁrez \\ Hospital Universitario San Ignacio, Colombia
}

a Autor de correspondencia. Correo electrónico: gami8203@yahoo.com

Cómo citar: Mosquera-Klinger G, Mayo-Patiño M, Suárez Y. Paracentesis: datos sobre la correcta utilización. Univ. Med. 2018;59(1): 53-60. doi: $\quad$ https://doi.org/10.11144/Javeriana.umed

\section{RESUMEN}

La paracentesis es un procedimiento con fines diagnósticos en pacientes con ascitis de novo. Permite detectar la presencia de infección en pacientes con ascitis de larga data. En casos de ascitis a tensión, ascitis refractaria y disnea por restricción es ampliamente utilizada como terapia. El conocimiento general de este procedimiento y de los pormenores de su técnica son de gran importancia, ya que su utilización viene en aumento en los servicios de urgencias, hospitalización general e incluso, en la unidad de cuidados intensivos. Teniendo en cuenta lo anterior, se hace hincapié en la correcta utilización de esta técnica por parte del personal médico. Métodos: Se realizó una búsqueda de la literatura científica en las bases de datos Pubmed y SciELO de artículos con una estrategia definida, limitada a idiomas español e inglés, utilizando una combinación de términos libres y MeSH que incluyeron paracentesis, ascitis, cirrosis hepática y malignidad.

Palabras clave

paracentesis; ascitis; cirrosis hepática.

\begin{abstract}
Paracentesis is a diagnostic procedure used in patients with newonset ascites. It can detect the presence of infection in patients with longstanding ascites. In cases of tense ascites, refractory ascites or restriction dyspnea it is widely used for therapeutical purposes. General knowledge of this procedure is of great importance as well as a detailed understanding of the technique, because its use is increasing in the emergency department, general hospitalization, and even in the intensive care unit. Taking all this into consideration we aim to emphasize on the proper use of this technique by the medical staff.

Keywords

paracentesis; ascites; liver cirrosis; malignancies.
\end{abstract}




\section{Introducción}

El objetivo de este artículo es hacer una revisión acerca de la paracentesis, un procedimiento diagnóstico y terapéutico de uso muy frecuente en los servicios de urgencias y hospitalización. La debe llevar a cabo personal médico entrenado, pues así tiene muy pocas posibilidades de complicaciones. El propósito último es proveer el mejor manejo posible en los pacientes que se someterán a paracentesis. Las anotaciones y recomendaciones están basadas en la mejor evidencia científica disponible y en el juicio clínico de expertos en el tema.

Decidimos hacer este artículo de revisión a partir de la experiencia que tuvimos en la realización de una guía institucional en el Hospital Universitario San Ignacio acerca de la paracentesis. Es un procedimiento sencillo que se hace con regularidad en los servicios de urgencias, hospitalización general $\mathrm{y}$, de acuerdo con algunas consideraciones, en la unidad de cuidados intensivos. Sus posibles complicaciones son escasas, pero ocasionalmente son tan graves que pueden generar mayor morbimortalidad, estancia hospitalaria y costos de atención.

La paracentesis abdominal y el apropiado análisis del líquido ascítico es, de algún modo, el método más rápido y costo-efectivo para el diagnóstico de las causas de la ascitis. En la atención actual de enfermedades de alta complejidad y por el aumento de la sobrevida global, cada vez más llegan pacientes con enfermedades que requieren este tipo de intervención (enfermedad hepática avanzada, ascitis por cáncer avanzado, etc.), por lo que es preciso tener conocimientos claros, así como unos datos que sirvan como guía en la práctica cotidiana, para aminorar las complicaciones relacionadas con el procedimiento.

La paracentesis fue descrita por primera vez por Salomón, a principios de siglo XX. En sus inicios se utilizaban agujas de gran tamaño, con lo cual había más complicaciones. Posteriormente, se introdujo el uso de diuréticos que incrementaron el manejo médico de la ascitis. Por lo anterior, estuvo en desuso en 1950, pero reapareció en 1980, con la publicación de artículos que evidenciaban que se trata de un procedimiento seguro $(1,2)$. En la actualidad este es común en todos los servicios de urgencias.
La paracentesis es una herramienta diagnóstica de gran importancia para pacientes con ascitis de novo, para determinar la etiología, y en pacientes con ascitis de larga data, para detectar la presencia de infección $(3,4)$.

Adicionalmente, tiene indicación terapéutica en el contexto de pacientes con dificultad respiratoria por restricción abdominal y para mejorar el malestar abdominal en pacientes con ascitis a tensión (en el contexto de enfermedad hepática terminal, la ascitis por cáncer principalmente en cáncer de ovario, de páncreas, estómago, colon, seno y variedad de sarcomas y linfomas).

\section{Indicaciones y contraindicaciones}

\section{Indicaciones}

Diagnóstica. En pacientes con ascitis preexistente, en quienes se sospecha peritonitis bacteriana espontánea. Pacientes adultos con ascitis de novo de etiología no clara. Se estudia el líquido ascítico (tabla 1) con el fin de evaluar sus características y determinar si es por hipertensión portal, cáncer, infección, pancreatitis o algún otro proceso (5).

\section{Tabla 1}

Datos de laboratorio del líquido ascitico

\begin{tabular}{|l|l|}
\hline Rutinarios & $\begin{array}{l}\text { Recuento celular y diferencial } \\
\text { Albúmina } \\
\text { Proteínas totales }\end{array}$ \\
\hline Opcionales & $\begin{array}{l}\text { Cultivo en botella de hemocultivo } \\
\text { Glucosa } \\
\text { Deshidrogenasa láctica } \\
\text { Amilasa } \\
\text { Tinción de Gram }\end{array}$ \\
\hline Inusuales & $\begin{array}{l}\text { Frotis y cultivo para bacilos acidorresistentes } \\
\text { Citología } \\
\text { Triglicéridos } \\
\text { Bilirrubina }\end{array}$ \\
\hline De poca utilidad & $\begin{array}{l}\text { pH } \\
\text { Lactato } \\
\text { Colesterol } \\
\text { Fibronectina } \\
\text { Glicosaminoglicanos }\end{array}$ \\
\hline
\end{tabular}

Fuente: adaptado de Runyon (9).

Algunos expertos recomiendan una paracentesis diagnóstica en todo paciente con ascitis y cirrosis que requiera ser hospitalizado, puesto que presenta 
una alta probabilidad de peritonitis bacteriana espontánea.

Terapéutica. Está indicada la paracentesis con extracción de altos volúmenes en pacientes con disnea por restricción, ascitis a tensión. Las paracentesis seriadas de altos volúmenes se pueden considerar en pacientes con ascitis intratable, ascitis refractaria o aquella que no responde al manejo con diuréticos (5).

\section{Contraindicaciones absolutas}

No hay contraindicaciones absolutas para hacer una paracentesis (6).

\section{Contraindicaciones relativas}

Hematoma en la pared abdominal o infecciones cutáneas en el sitio escogido para la punción; así mismo, lesiones traumáticas de la pared abdominal, trombocitopenia o alteración en los tiempos de coagulación (5).

\section{Precauciones (casos especiales)}

Pacientes embarazadas, historia de cirugía abdominal reciente o múltiples intervenciones en el abdomen, obstrucción intestinal, coagulopatía, ascitis tabicada, enfermedad renal crónica estadio 5 , infecciones en la piel en el sitio de la punción, visceromegalias y adherencias abdominales.

\section{Personal equipos e insumos necesarios}

Recurso técnico. Gafas protectoras, guantes estériles, bata, gorro, máscara esterilizada, solución desinfectante (povidona yodada), gasas esterilizadas $4 \times 4$, solución anestésica con epinefrina o sin esta, aguja calibre 25 , jeringa de $10 \mathrm{ml}$, agujas calibre 18 o aguja espinal, jeringa de $50 \mathrm{ml}$ para recoger el líquido ascítico, vendas y llaves de 3 vías.

Opcionales: sistemas de recogida de sangre si se utilizan frascos de vacío, frascos de hemocultivos, contenedor esterilizado de especimen para citología.
La paracentesis se debe realizar en una sala especial de procedimientos.

Recurso humano. Siempre debe hacerse por parte de personal médico. En casos especiales, podrá considerarse la punción bajo visión ecográfica y bajo la vigilancia del médico más experimentado en el turno.

\section{Técnica}

Preparación. Se explica el procedimiento al paciente, sus riesgos y sus beneficios, o a sus familiares o acudientes. Se debe obtener el consentimiento informado del procedimiento.

Es mandatorio tener en cuenta vaciar la vejiga del paciente. Si este es incapaz de orinar voluntariamente, se deberá pasar una sonda vesical con fines de evacuar la vejiga. En caso de obstrucción intestinal, se pasa una sonda nasogástrica.

Areas recomendadas para la paracentesis. Usualmente se punciona la unión entre los dos tercios mediales con el tercio lateral, de una línea imaginaria trazada entre la espina iliaca anterosuperior y el ombligo en ambos lados. La elección del lado es de preferencia y de la experiencia del médico que va a realizar el procedimiento. Las complicaciones en ambos lados tienen una incidencia similar. La más frecuente del lado derecho es la punción del ciego; en algunas ocasiones perforación de este.

En pacientes que presenten circulación colateral, se puede realizar la punción en un reparo anatómico alternativo, habitualmente $2 \mathrm{~cm}$ por debajo del ombligo por la línea media (línea alba) entre la sínfisis púbica y el ombligo (6,7) (figura 1 ). 


\section{Figura 1}

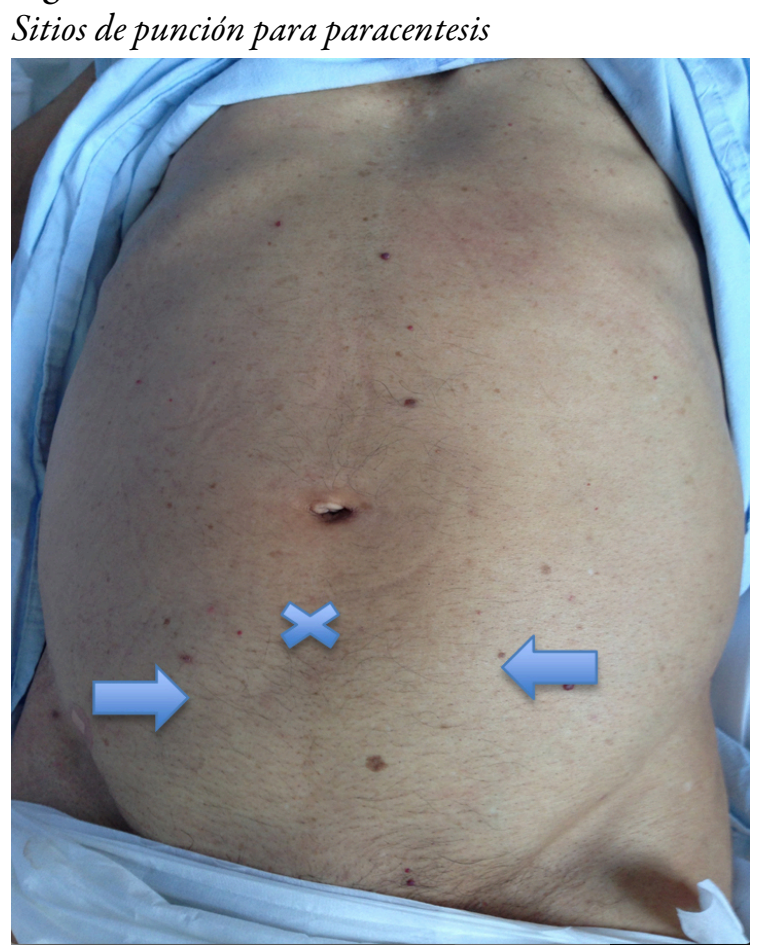

\section{Preparación del paciente}

Limpiar la piel alrededor del reparo anatómico escogido para la punción. Aplicar la solución antiséptica y dejar secar. Colocar paños esterilizados o el campo estéril, infiltrar de 2 a $5 \mathrm{ml}$ de anestesia local subcutánea y a lo largo del trayecto de inserción de la aguja. Posteriormente, esperar 3 o 5 min para lograr el efecto anestésico local. Se debe realizar una punción única, perpendicular a la pared abdominal, con aguja seleccionada aspirando continuamente hasta obtener retorno del líquido ascítico. En caso de no retorno del líquido se recomienda una nueva punción en un sitio diferente; en caso de ser negativo, se suspende el procedimiento. Si se sospecha punción vascular, se retira el equipo de punción y se hace una compresión sobre el sitio.

\section{Análisis del líquido peritoneal}

En sospecha de ascitis no complicada por cirrosis, únicamente se solicitan los exámenes de laboratorio de rutina (recuento celular y diferencial, albúmina y proteínas totales) a partir de la muestra de líquido ascítico obtenido (tabla 1). Si los resultados de los estudios anteriores son anormales, se puede tomar una muestra adicional y considerar más estudios. Algunos autores sugieren que con un adecuado almacenamiento de la muestra es posible estudiarla varios días después de la toma. Sin embargo, en la mayoría de los pacientes no son necesarios estudios adicionales, dado que la ascitis no complicada por cirrosis es la más frecuente (8).

Se requiere tomar la muestra en un tubo seco para medir el gradiente de albúmina y en un tubo con ácido etilendiaminotetraacético (EDTA) para conteo celular y recuento diferencial (tubo tapa lila) (5).

Si se sospecha infección del líquido ascítico (fiebre, dolor abdominal, encefalopatía, acidosis, hipotensión o hipotermia), está indicado el cultivo aerobio y anaerobio del líquido ascítico en una botella de hemocultivo (8). Según la clínica del paciente, se pueden solicitar estudios adicionales para confirmar el diagnóstico (tabla 2).

\section{Tabla 2}

\section{Características del líquido ascítico}

\begin{tabular}{|l|l|}
\hline Test y tipo de tubo & \multicolumn{1}{c|}{ Interpretación de resultados } \\
\hline Proteínas totales & $\begin{array}{l}\text { Valores } \geq 1 \mathrm{~g} / \mathrm{dl} \text { sugiere peritonitis secundaria, en vez de } \\
\text { peritonitis bacteriana espontánea }\end{array}$ \\
\hline Deshidrogenasa láctica & $\begin{array}{l}\text { Valores inferiores al límite superior de los valores séricos } \\
\text { normales sugieren peritonitis secundaria, en vez de } \\
\text { peritonitis bacteriana espontánea }\end{array}$ \\
\hline Glucosa & $\begin{array}{l}\text { Valores }<50 \mathrm{mg} / \text { dl sugieren peritonitis secundaria, en vez } \\
\text { de peritonitis bacteriana espontánea }\end{array}$ \\
\hline $\begin{array}{l}\text { Antígeno } \\
\text { carcinoembrionario }\end{array}$ & Valores $>5 \mathrm{ng} / \mathrm{ml}$ sugieren perforación de la víscera hueca \\
\hline Fosfatasa alcalina & Valores $>240$ U/L sugieren perforación de la víscera hueca \\
\hline Amilasa & $\begin{array}{l}\text { Valores marcadamente elevados (> 2000 U/L o } 5 \text { veces las } \\
\text { concentraciones séricas) en pacientes con ascitis } \\
\text { pancreática o perforación de la víscera hueca }\end{array}$ \\
\hline Triglicéridos & Valores $>200$ mg sugieren ascitis quilosa \\
\hline & \multicolumn{2}{|c|}{ Tubo estéril } \\
\hline Citología & $\begin{array}{l}\text { La sensibilidad se incrementa si se toman 3 muestras } \\
\text { seriadas rápidamente procesadas }\end{array}$ \\
\hline $\begin{array}{l}\text { Cultivo para } \\
\text { micobacterias }\end{array}$ & Sensibilidad del 50\% \\
\hline
\end{tabular}

Fuente: adaptado de Thomsen Paracentesis articles: NEJM.org (5)

Las concentraciones de proteínas totales, deshidrogenasa láctica y glucosa en el líquido ascítico permiten ayudar a diferenciar entre una peritonitis bacteriana espontánea y una secundaria. Son altamente sugestivos de peritonitis secundaria aquellos valores en el líquido ascítico de proteínas 
totales mayores o iguales a $1 \mathrm{~g} / \mathrm{dl}$, una glucosa menor de $50 \mathrm{~g} / \mathrm{dl}$ y valores de deshidrogenasa láctica inferiores al límite superior de los valores séricos normales. El cultivo de la muestra provee la confirmación del diagnóstico, pero este solo está indicado en pacientes sintomáticos. Aquellos pacientes con diagnóstico de peritonitis bacteriana espontánea deben ser tratados con cefalosporinas de tercera generación y con albúmina intravenosa (5).

Han mostrado una alta sensibilidad diagnóstica para perforación de víscera hueca las cantidades de líquido ascítico de antígeno carcinoembrionario mayores a $5 \mathrm{ng} / \mathrm{ml}$ o de fosfatasa alcalina mayor a $240 \mathrm{U} / \mathrm{L}$ (8). Valores marcadamente elevados de amilasa en el líquido (> $2000 \mathrm{U} / \mathrm{L}$ o 5 veces los niveles séricos) son sugestivos de ascitis pancreática o de perforación de la víscera hueca. Así mismo, triglicéridos mayores de $200 \mathrm{mg}$ en el líquido ascítico sugieren ascitis quilosa.

El frotis y el cultivo para micobacterias y la citología son laboratorios de alto costo, por lo cual deben solicitarse únicamente si existe una alta probabilidad pre test de la patología en consideración. Los pacientes con carcinomatosis peritoneal usualmente tienen un antecedente de tumor primario gástrico, mamario, pancreático o de colon. La citología del líquido ascítico es positiva solo en el contexto de carcinomatosis peritoneal con una sensibilidad del $96,7 \%$ con tres muestras seriadas.

La sensibilidad del frotis para micobacterias es del $0 \%$, y la del cultivo es del $50 \%$. Se indica la realización de frotis y cultivo para micobacterias en pacientes con alto riesgo para peritonitis tuberculosa, como es el caso de inmigrantes de áreas endémicas o pacientes con síndrome de inmunodeficiencia adquirirla. El método más rápido y preciso para su diagnóstico es la laparoscopia y la biopsia con cultivo para micobacterias (8).

\section{Gradiente de albúmina}

La medición del gradiente de albumina (sérico/ ascítico) es una estrategia que permite determinar si se trata de un líquido hipertensivo o no. Este gradiente se calcula restando las concentraciones de albúmina del líquido ascítico de la albúmina sérica: albúmina sérica - albúmina del líquido ascítico. Valores mayores o iguales a $1,1 \mathrm{~g} / \mathrm{dl}$ indican etiología hipertensiva con un $97 \%$ de precisión y, usualmente, responden de modo favorable a la restricción de sal y al manejo con diuréticos. Aquellos pacientes con hipertensión portal y ascitis de causa secundaria también presentan un gradiente de albúmina mayor o igual a $1,1 \mathrm{~g} / \mathrm{dl}$.

Los valores menores de $1,1 \mathrm{~g} / \mathrm{dl}$ corresponden a otras causas (tabla 3 ), no cursan con hipertensión portal y no responden a manejo con diurético ni con restricción de sal (excepto en caso de síndrome nefrótico) (8).

\section{Tabla 3}

\section{Diagnósticos diferenciales según el gradiente de} albúmina sérico y del líquido ascítico

\begin{tabular}{|c|c|}
\hline Gradiente $\geq 1,1 \mathrm{~g} / \mathrm{dl}$ (hipertensivo) & Gradiente $<1,1 \mathrm{~g} / \mathrm{dl}$ (no hipertensivo) \\
\hline $\begin{array}{l}\text { Cirrosis } \\
\text { Hepatitis alcohólica } \\
\text { Ascitis de origen cardiaca } \\
\text { Trombosis de la vena porta } \\
\text { Síndrome de Budd-Chiari } \\
\text { Metástasis hepáticas }\end{array}$ & $\begin{array}{l}\text { Carcinomatosis peritoneal } \\
\text { Peritonitis tuberculosa } \\
\text { Ascitis pancreática } \\
\text { Ascitis biliar } \\
\text { Síndrome nefrótico } \\
\text { Serositis }\end{array}$ \\
\hline \multicolumn{2}{|c|}{ Fuente: adaptado de Thomsen } \\
\hline
\end{tabular}

En casos de evacuaciones grandes, mayores de $4000 \mathrm{ml}$ de líquido ascítico, varios expertos recomiendan considerar la infusión de 6 a $8 \mathrm{~g}$ albúmina por cada litro de líquido ascítico extraído. Sin embargo, esto sigue siendo controversial, dado que el uso de albúmina es de alto costo y aún no hay suficiente evidencia acerca de los beneficios en la sobrevida de los pacientes (5).

\section{Complicaciones}

Las complicaciones se estiman entre el $1 \%$ y el $5 \%$, y rara vez son graves. Las probables complicaciones graves incluyen hematoma de la pared abdominal, hemoperitoneo, perforación intestinal que requiera cirugía de emergencia (laparotomía exploratoria), fuga persistente de líquido ascítico y afectación hemodinámica sistémica. La mayor parte de las complicaciones son locales, y de estas las más frecuentes son las hemorrágicas. Muchos pacientes que se someten a paracentesis son cirróticos, que frecuentemente cursan con anomalías plaquetarias y en los tiempos de coagulación (en especial INR). En este grupo de pacientes no se recomienda hacer 
transfusiones de plaquetas o de factores antes del procedimiento a menos que el nivel de plaquetas sea crítico (menor de 20.000). En estos casos especiales se debe considerar la paracentesis con abordaje en la línea media infraumbilical (línea alba). Esta zona está desprovista de vasos sanguíneos, lo que reduce la posibilidad de complicaciones (9).

En un estudio de 4729 paracentesis, los investigadores informaron que 8 de las 9 complicaciones hemorrágicas que se presentaron ocurrieron en pacientes con insuficiencia renal. Tal vez la anomalía cualitativa de plaquetas en ese contexto predispone a más hemorragia (8). Parece que la incidencia de complicaciones hemorrágicas en el grupo de pacientes sin defectos cualitativos de las plaquetas es menor.

Aunque la hemorragia es una complicación potencial de la paracentesis, la administración de productos sanguíneos profilácticos, como plasma fresco congelado, no parece tener justificación para usarlo (10). En pacientes sobreanticoagulados farmacológicamente (warfarina con INR mayor del rango terapéutico) si se recomienda llevar a rango terapéutico previo al procedimiento. El médico decidirá la corrección según el contexto clínico.

Las pruebas rutinarias de coagulación no reflejan el riesgo de hemorragia en pacientes con cirrosis; estos podrían tener pruebas normales a causa de las deficiencias equilibradas de factores procoagulantes y anticoagulantes $(11,12,13)$. Los riesgos y costos de las transfusiones profilácticas pueden exceder los beneficios; por esta razón no recomendamos su uso rutinario en estos pacientes.

En relación con paracentesis en pacientes con una historia previa conocida de trombocitopenia, se sugiere la realización de un hemograma y evitar la paracentesis si el conteo de plaquetas es inferior a 20.000 células $/ \mathrm{mm}^{3}$. En un estudio de 1100 paracentesis de grandes volúmenes no se presentaron complicaciones hemorrágicas a pesar de los siguientes puntos:

1. No se colocaron transfusiones profilácticas.

2. El conteo plaquetario fue tan bajo como 19.000 células $/ \mathrm{mm}^{3}$; en el $54 \%$ fueron menores de 50.000 células $/ \mathrm{mm}^{3}$.

3. El INR en el $75 \%$ de los pacientes fue mayor de 1,5 , y en el $26 \%$ fue mayor de 2 (14).
El sangrado asociado a la paracentesis ocurre en menos de uno de cada mil procedimientos. Se han reportado casos de hemoperitoneo espontáneo secundario a várices mesentéricas en paracentesis de grandes volúmenes (mayor de $4000 \mathrm{ml}$ ). Esta condición tiene una alta mortalidad $(2,7,12)$. Se ha descrito una complicación vascular adicional, que es el seudoaneurisma de la arteria epigástrica inferior (15). Hay reportes recientes de casos que demuestran la utilidad de la ecografía abdominal en la localización de la arteria epigástrica inferior, con la disminución potencial de esta complicación (16).

La evidencia indica que las complicaciones hemorrágicas se pueden presentar de forma espontánea, por lo que se deben tener precauciones sobre el sitio de punción. A pesar del hecho de que en casi todos los estudios publicados sobre paracentesis se excluyeron los pacientes con peritonitis bacteriana espontánea, insuficiencia renal, encefalopatía hepática grave, trombocitopenia, hipotensión arterial o ictericia severa, no hay hallazgos de que estas complicaciones se deban considerar contraindicaciones para paracentesis en la práctica clínica $(17,18)$.

La disfunción circulatoria puede ocurrir después del drenaje de grandes volúmenes en la paracentesis, y esta se asocia con hipotensión, hiponatremia e incremento de catecolaminas y reninas plasmáticas $(5,19,20,21)$.

Si el líquido ascítico es difícil de localizar en el examen físico (por ejemplo, por obesidad) o en pacientes con múltiples intervenciones quirúrgicas abdominales previas, puede utilizarse ultrasonografía abdominal. Esta última es útil para la localización y punción del líquido, así como para evitar puncionar estructuras como el bazo y el hígado. La paracentesis es un procedimiento seguro, con pocas complicaciones, por lo que puede realizarse por un médico entrenado.

\section{Cuidados después de procedimiento}

Después de terminado el procedimiento, debe vendarse el lugar de entrada de la paracentesis, a fin de minimizar el riesgo de infecciones locales y drenaje pospunción. En caso de pacientes con ascitis a tensión, puede ocurrir la filtración de líquido a 
través de la pared. Se recomienda el cambio de apósitos regularmente. La cinta adhesiva es útil para evitar la fuga de líquido sobre la ropa y cama del paciente.

El paciente debe estar en reposo unos minutos después de la paracentesis para minimizar el riesgo de caídas en relación con hipotensión postural por la pérdida de líquidos.

\section{Conflictos de interés}

Los autores de la revisión declaramos no tener conflictos de interés.

\section{Referencias}

1. 1. Runyon B. Paracentesis of ascites fluid: A safe procedure. Arch Intern Med. 1986;146:2259-61.

2. 2. Pinto PC, American J, Reynolds TB. Large-volume paracentesis in the nonedematous patient with tense ascites: Its effect on in intravascular volume. Hepatology. 1988;8(2):207-10.

3. 3. Pinzello G, Simonetti RG, Craxi A, et al. spontaneous bacterial peritonitis: a prospective investigation in predominately nonalcoholic cirrhotic patients. Hepatology. 1983;3 (4):545-9.

4. 4. Koffel KK, Reed JS. The technique of abdominal paracentesis. J Crit Ill. 1986;1:45-50.

5. 5. Thomsen $T$, Shaffer $R$, White $B$, Setnik G. Paracentesis. N Engl J Med. 2006;355:e21.

6. 6. Reichman EF, Simon RR. Emergency medicine procedures. New York: McGraw Hill; 2003.

7. 7. Runyon B. Care of patients with ascites. N Engl J Med. 1994;330(5):337-341.

8. 8. Runyon B. Management of adult patients with ascites caused by cirrhosis. Hepatology. 1998;27(1):264-72.

9. 9. Pache I, Bilodeau M. Severe hemorrhage following abdominal paracentesis for ascites in patients with liver failure. Aliment Pharmacol Ther. 2005;21:525-9.

10. 10. Mannucci PM. Abnormal hemostasis tests and bleeding in chronic liver disease: Are they related? No. J Thromb Haemost. 2006;4:721-3.

11. 11. Caldwell SH, Hoffman $M$, Lisman T, Macik BG, Northup PG, Reddy KR, et al. Coagulation disorders and hemostasis in liver disease: pathophysiology and critical assessment of current management. Hepatology. 2006;44:1039-46.

12. 12. Arnold C, Klaus H, Blum HE, et al. Acute hemoperitoneum after largevolume paracentesis. Gastroenterology. 1997;113(3):978-82.

13. 13. Grabau CM, Crago SF, Hoff LK, Simon JA, Melton CA, Ott BJ, et al. Performance standards for therapeutic abdominal paracentesis. Hepatology. 2004;40:484-8.

14. 14. McVay PA, Toy PT. Lack of increased bleeding after paracentesis and thoracentesis in patients with mild coagulation abnormalities. Transfusion. 1991;31:164-71.

15. 15. Lam EY, McLafferty RB, Taylor $\mathrm{LM}$, et al. Inferior epigastric artery pseudoaneurysm: A complication of paracentesis. J Vasc Surg 1998;28(3):566-9.

16. 16. Stone J, Moak J. Feasibility of sonographic localization of the inferior epigastric artery before ultrasound-guided paracentesis: a case series. Am J Emerg Med. 2015;33(12):1795-8. doi: https:// doi.org/10.1016/j.ajem.2015.06.067

17. 17. Moore KP, Wong K, Gines P, et al. The management of ascites in cirrhosis: 
Report on the Consensus Conference of the International Ascites Club. Hepatology. 2003;38(1):258-66.

18. 18. Sandhu BS, Sanyal AJ. Management of ascites in cirrhosis. Clin Liver Dis. 2005;9:715-32.

19. 19. Thomsen T, Shaffer R, White B, Setnik G. Paracentesis. N Engl J Med. 2006;355:e21.

20. 20. Runyon BA. AASLD Practice Guidelines Committee. Management of adult patients with ascites due to cirrhosis: an update. Hepatology. 2009;49:2087-2107.

21. 21. Insuasty MR, Restrepo JC. Paracentesis evacuante en pacientes cirróticos con ascitis a tensión: revisión del tema. Iatrea. 2010;23(3):259-67.

\section{Notas}

* Artículo de revisión. 